\title{
A Multilevel Finite Element Variational Multiscale Method for Incompressible Navier-Stokes Equations Based on Two Local Gauss Integrations
}

\author{
Yamiao Zhang, Biwu Huang, Jiazhong Zhang, and Zexia Zhang \\ School of Energy and Power Engineering, Xi'an Jiaotong University, Xi'an 710049, China \\ Correspondence should be addressed to Jiazhong Zhang; jzzhang@mail.xjtu.edu.cn
}

Received 22 May 2017; Revised 23 July 2017; Accepted 5 September 2017; Published 26 November 2017

Academic Editor: Xesús Nogueira

Copyright (c) 2017 Yamiao Zhang et al. This is an open access article distributed under the Creative Commons Attribution License, which permits unrestricted use, distribution, and reproduction in any medium, provided the original work is properly cited.

\begin{abstract}
A multilevel finite element variational multiscale method is proposed and applied to the numerical simulation of incompressible Navier-Stokes equations. This method combines the finite element variational multiscale method based on two local Gauss integrations with the multilevel discretization using Newton correction on each step. The main idea of the multilevel finite element variational multiscale method is that the equations are first solved on a single coarse grid by finite element variational multiscale method; then finite element variational multiscale approximations are generated on a succession of refined grids by solving a linearized problem. Moreover, the stability analysis and error estimate of the multilevel finite element variational multiscale method are given. Finally, some numerical examples are presented to support the theoretical analysis and to check the efficiency of the proposed method. The results show that the multilevel finite element variational multiscale method is more efficient than the onelevel finite element variational multiscale method, and for an appropriate choice of meshes, the multilevel finite element variational multiscale method is not only time-saving but also highly accurate.
\end{abstract}

\section{Introduction}

The finite element method is one of the most general techniques for incompressible Navier-Stokes equations. However, in numerical simulations of the incompressible NavierStokes equations at higher Reynolds number, the standard Galerkin finite element method is often failed due to the domination of convection term [1-3]. This defect can be overcome to some extent by a variety of stabilizing techniques, including Galerkin least square (GLS) method [4, 5], streamline-upwind Petrov-Galerkin (SUPG) method [6], defect-correction method $[7,8]$, local projection stabilization (LPS) method [9], discontinuous Galerkin method [10-12], and variational multiscale (VMS) method. Among them, the VMS method is one of some successful stabilized methods that was originally introduced and developed by Hughes et al. [13-16] as a technique in the construction of subgridscale model in large eddy simulation for incompressible and compressible flows. Subsequently, it was also used as a subgrid stabilized technique to improve the accuracy of the solution which was obtained by the Galerkin finite element approximation for the convection dominated problems [17-19]. In the VMS method, the governing equations are rewritten as a coupled system with two types of scales: large scales and small scales. Furthermore, according to Collis [20], the governing equations can also be rewritten as a coupled system with three types of scales: resolved large scales, resolved small scales, and unresolved scales. As solving the above coupled system by finite element method, the traditional Galerkin approximation can be improved by adding an asymptotically consistent artificial diffusion term on the subgrid scales. Under the circumstance, it is necessary to define an appropriate operator, function spaces, and some additional dependent variables. Recently, a finite element VMS method based on two local Gauss integrations was proposed and developed by the authors of [21-25]. This finite element VMS method is simpler than the common finite element VMS method, 
which does not need additional dependent variables and the projection operator but keeps the same efficiency.

However, when solving nonlinear partial differential equation directly using this finite element VMS method based on two local Gauss integrations, it needs too much computing time, especially for conditions requiring a relatively fine-grid discretization. A treatment for this problem is the multilevel discretization. The multilevel method is a wellestablished and efficient method for solving nonlinear partial differential equations. Some classical multilevel methods can be found in [26-32]. The main idea of the multilevel method is to solve the nonlinear equations first on a coarse grid and then solve a linearized problem corresponding to this nonlinear governing equation on a succession of refined grids. Hence, the multilevel method can save much computing time compared with the one-level method. Nevertheless, there is a shortcoming for these classical multilevel methods in discretization of the convection dominated Navier-Stokes equations; that is, the nonlinear Navier-Stokes equations need to be first solved on the coarsest grid by standard Galerkin finite element method. This may lead to numerical oscillations in the coarse grid approximation and transmit remarkable error to the subsequent refined grid numerical approximation and hence cannot yield a correct approximation.

In this paper, by combining the multilevel discretization strategy with the finite element VMS method based on two local Gauss integrations, we propose a multilevel finite element VMS method for the convection dominated NavierStokes equations. In this method, the nonlinear governing equations are first solved on a single coarse grid by finite element VMS method based on two local Gauss integrations, and then finite element VMS approximations are generated on a succession of refined grids using Newton correction on each step by solving a linearized problem corresponding to the nonlinear governing equations. Compared with the classical multilevel methods, the proposed method can solve the convection dominated Navier-Stokes equations more accurately. While compared with the one-level finite element VMS method, the proposed method can save plenty of computing time in fine-grid discretization.

It is noted that a multilevel VMS method has been reported in [33]. However, there are two essential differences between the proposed method and the one studied in [33]. First, the proposed VMS method in this paper is based on two local Gauss integrations while the one in [33] is the residualbased VMS method. Second, the purpose and the refinement strategy are different. The meshes in the proposed method are global refinement in domains with a Newton correction at each level to increase the efficiency of numerical scheme based on the finite element discretization, while the meshes in [33] are local adaptive refinement in domains via a clear hierarchy, multilevel $h p$-formulation for further developing the finite cell methodology. Moreover, the two-level case of the proposed method is also different from those in $[34,35]$. Compared with the two-level projection-based VMS method in [34], the proposed method in this paper does not need additional dependent variables and the projection operator, so it is simpler. Compared with the two-level VMS method in
[35], the proposed method uses Newton correction to obtain a better approximation than the Stokes correction used in [35]. And what is more, the theoretical analysis and results of [35] are limited to the small data condition, while the condition in this paper is no longer needed, so that the proposed method has a wider effective range than the method in [35].

This paper is organized as follows. In Section 2, the Navier-Stokes equations, notations, and well-known results used throughout this paper are given. In Section 3, the finite element VMS method is introduced. Then, the multilevel finite element VMS method is presented and the corresponding stability and convergence error are analyzed in Section 4. In Section 5, three numerical examples are given to verify the theoretical analysis. Finally, conclusions are drawn in Section 6.

\section{Preliminaries}

In this paper, the following steady Navier-Stokes equations will be considered:

$$
\begin{array}{rc}
-v \Delta u+(u \cdot \nabla) u+\nabla p=f & \text { in } \Omega, \\
\operatorname{div} u=0 & \text { in } \Omega, \\
u=0 & \text { on } \partial \Omega,
\end{array}
$$

where $\Omega$ is a bounded domain in $R^{2}$ with a Lipschitz continuous boundary $\partial \Omega, u=\left(u_{1}, u_{2}\right)$ the fluid velocity, $p$ the pressure, $f$ the prescribed body force, and $v>0$ the kinematic viscosity which represents the inverse of Reynolds number Re. For the mathematical setting of problem (1), the following Hilbert spaces are needed:

$$
\begin{aligned}
& X=H_{0}^{1}(\Omega)^{2}, \\
& M=L_{0}^{2}(\Omega)=\left\{q \in L^{2}(\Omega): \int_{\Omega} q d x=0\right\} .
\end{aligned}
$$

The norm and the inner product in $L^{2}(\Omega)$ are denoted by $\|\cdot\|_{0}$ and $(\cdot, \cdot)$, respectively. $\|\cdot\|_{k}$ is the norm of the Sobolev space $H^{k}(\Omega)$ or $H^{k}(\Omega)^{2}, k=1,2$. Using the Poincaré inequality, the norm in space $X$ equipped with $\|\nabla \cdot\|_{0}$ and $\|\cdot\|_{1}$ can be considered to be equivalent. We define the continuous bilinear forms $a(\cdot, \cdot)$ and $d(\cdot, \cdot)$, and the trilinear term $b(\cdot, \cdot, \cdot)$ by

$$
\begin{aligned}
& a(u, v)=(\nabla u, \nabla v), \quad \forall u, v \in X, \\
& d(v, q)=(q, \operatorname{div} v), \quad \forall(v, q) \in(X \times M), \\
& b(u, v, w)=((u \cdot \nabla) v, w)+\frac{1}{2}((\nabla \cdot u) v, w) \\
&=\frac{1}{2}((u \cdot \nabla) v, w)-\frac{1}{2}((u \cdot \nabla) w, v), \\
& \forall u, v, w \in X,
\end{aligned}
$$


respectively. The trilinear term $b(\cdot, \cdot, \cdot)$ satisfies the following estimates [36]:

$$
\begin{aligned}
b(u, v, v) & =0, \quad \forall u, v, w \in X, \\
|b(u, v, w)| & \leq N\|\nabla u\|_{0}\|\nabla v\|_{0}\|\nabla w\|_{0}, \quad \forall u, v, w \in X,
\end{aligned}
$$

where $N$ is a positive constant depending only on $\Omega$. Subsequently, $c$ and $k$ (with a subscript) will denote a positive constant which may stand for different values at its different occurrences, respectively.

With the above notations, the variational formulation of problem (1) reads as follows: find $(u, p) \in X \times M$ for all $(v, q) \in$ $X \times M$ satisfying

$$
v a(u, v)-d(v, p)+d(u, q)+b(u, u, v)=(f, v) .
$$

The nonsingular solution of (1) is defined as follows [37]: $u$ is a nonsingular solution of $(1)$ if there is a constant $\sigma(u, v)>0$ such that

$$
\inf _{v \in V} \sup _{w \in V} \frac{v a(v, w)+b(u, v, w)+b(v, u, w)}{\|v\|_{1}\|w\|_{1}} \geq \sigma(u, v) .
$$

Here $V=\{v \in X:(\nabla \cdot v, q)=0, \forall q \in M\}$. If $u$ is a nonsingular solution of the Navier-Stokes equations, $u$ satisfies the estimate $[37,38]$

$$
\|u\|_{1} \leq v^{-1}\|f\|_{-1}, \quad\|f\|_{-1}=\sup _{v \in X, v \neq 0} \frac{|(f, v)|}{\|v\|_{1}} .
$$

Lemma 1 is also needed; more details of it can be found in $[39,40]$.

Lemma 1. Suppose that

$$
\inf _{q \in M_{v \in X}} \frac{d(v, q)}{\|q\|_{0}\|v\|_{1}} \geq \beta>0
$$

and that the bilinear form $c(u, v)$ satisfies

$$
\inf _{u \in V} \sup _{v \in V} \frac{c(u, v)}{\|u\|_{1}\|v\|_{1}} \geq \alpha_{0}>0 .
$$

Then the continuous bilinear form $F:(X, M) \times(X, M) \rightarrow R$ defined by

$$
F[(u, p) ;(v, q)]:=c(u, v)-d(v, p)+d(u, q)
$$

satisfies the inf-sup condition on the product space $(X, M) \times$ $(X, M)$, which means that there exists a constant $\gamma^{*}=$ $\gamma^{*}\left(\alpha_{0} ; \beta ;\|c(\cdot, \cdot)\|\right)>0$ such that

$$
\inf _{(u, p) \in(X, M)} \sup _{(v, q) \in(X, M)} \frac{F[(u, p) ;(v, q)]}{\|(u, p)\|\|\|(v, q) \|} \geq \gamma^{*} .
$$

For the finite element discretization, let $K_{h}$ be the regular triangulations of the domain $\Omega$. The mesh defined by $h=\max _{\Omega_{e} \in K_{h}}\left\{\operatorname{diam}\left(\Omega_{e}\right)\right\}$ is a real-positive parameter tending to 0 . We choose the conforming velocity-pressure finite element space $\left(X_{h}, M_{h}\right) \subset(X, M)$ and consider the TaylorHood elements [41]

$$
\begin{aligned}
& X_{h} \\
& =\left\{v_{h} \in C^{0}(\Omega)^{2} \cap X:\left.v_{h}\right|_{\Omega_{e}} \in P_{2}\left(\Omega_{e}\right)^{2}, \forall \Omega_{e} \in K_{h}\right\}, \\
& M_{h} \\
& =\left\{q_{h} \in C^{0}(\Omega) \cap M:\left.q_{h}\right|_{\Omega_{e}} \in P_{1}\left(\Omega_{e}\right), \forall \Omega_{e} \in K_{h}\right\},
\end{aligned}
$$

where $P_{j}\left(\Omega_{e}\right)(j=1,2)$ is the space of $j$ th order polynomials on $\Omega_{e}$. The Taylor-Hood elements on both triangles and rectangles are proved to satisfy the following discrete inf-sup condition for compatibility of the velocity-pressure spaces:

$$
\inf _{q_{h} \in M_{h} \sup _{h} \in X_{h}} \frac{d\left(v_{h}, q_{h}\right)}{\left\|q_{h}\right\|_{0}\left\|v_{h}\right\|_{1}} \geq \beta>0 .
$$

\section{Finite Element Variational Multiscale Method}

The finite element VMS method proposed by John and Kaya $[19,42]$ for the steady Navier-Stokes equations reads find $\left(u_{h}, p_{h}\right) \in\left(X_{h}, M_{h}\right)$ and $g_{h} \in L_{h}$ for all $\left(v_{h}, q_{h}\right) \in\left(X_{h}, M_{h}\right)$ satisfying

$$
\begin{aligned}
& (v+\alpha) a\left(u_{h}, v_{h}\right)-\alpha\left(g_{h}, \nabla v_{h}\right)+b\left(u_{h}, u_{h}, v_{h}\right) \\
& -d\left(v_{h}, p_{h}\right)+d\left(u_{h}, q_{h}\right)=\left(f, v_{h}\right), \\
& \left(g_{h}-\nabla u_{h}, l_{h}\right)=0 \quad \forall l_{h} \in L_{h} .
\end{aligned}
$$

This system is determined by the choices of $L_{h}$ and $\alpha$. The stabilization parameter $0<\alpha<1$ acts only on the small scales which can be chosen as the scale of $O\left(h^{s}\right)(s \geq 1)$ in order to stabilize the convective term appropriately.

An orthogonal projection operator is defined as $\Pi$ : $L \rightarrow L_{h}$ which is $L^{2}$ orthogonal projection with the following properties:

$$
\begin{aligned}
&(\Pi \nabla u, v)=(\nabla u, v), \quad \forall u \in X, v \in L_{h}, \\
&\|\Pi \nabla v\|_{0} \leq C\|\nabla v\|_{0}, \quad \forall v \in X, \\
&\|(I-\Pi) \nabla v\|_{0} \leq C\|\nabla v\|_{0}, \quad \forall v \in X, \\
&\|(I-\Pi) \nabla v\|_{0} \leq C h\|v\|_{2}, \quad \forall v \in H^{2}(\Omega)^{2 \times 2} \cap X,
\end{aligned}
$$

where $C$ is a positive constant, $L=L^{2}(\Omega)$, and $L_{h} \subset L$ (for details, refer to [18]).

Based on the properties of the orthogonal projection operator $\Pi$, the finite element variational multiscale method 
proposed by Zheng et al. [21] is as follows: find $\left(u_{h}, p_{h}\right) \in$ $\left(X_{h}, M_{h}\right)$ for all $\left(v_{h}, q_{h}\right) \in\left(X_{h}, M_{h}\right)$ satisfying

$$
\begin{aligned}
& v a\left(u_{h}, v_{h}\right)-d\left(v_{h}, p_{h}\right)+d\left(u_{h}, q_{h}\right)+b\left(u_{h}, u_{h}, v_{h}\right) \\
& +G\left(u_{h}, v_{h}\right)=\left(f, v_{h}\right) .
\end{aligned}
$$

Here,

$$
\begin{aligned}
G & \left(u_{h}, v_{h}\right)=\alpha\left((I-\Pi) \nabla u_{h},(I-\Pi) \nabla v_{h}\right) \\
& =\alpha \sum_{\Omega_{e} \in \tau_{h}}\left\{\int_{\Omega_{e}, k} \nabla u_{h} \nabla v_{h} d x-\int_{\Omega_{e}, 1} \nabla u_{h} \nabla v_{h} d x\right\},
\end{aligned}
$$

where $\int_{\Omega_{e, i}}(\cdot) d x$ denotes an appropriate Gauss integral over $\Omega_{e}$ which is exact for polynomials of degree $i, i=1, k(k \geq$ 2).

From $[24,35]$, problem (16) has the following stability and error estimate.

Theorem 2. Suppose the finite element space $\left(X_{h}, M_{h}\right)$ satisfies the LBB condition (14). Let $(u, p) \in\left(H^{2}(\Omega)^{2} \cap X, H^{1}(\Omega) \cap M\right)$ be a nonsingular solution of (6). Then the approximate solution $\left(u_{h}, p_{h}\right)$ given by (16) satisfies

$$
\left\|u_{h}\right\|_{1} \leq v^{-1}\|f\|_{-1}
$$

and the following error estimate

$$
\left\|u-u_{h}\right\|_{1}+\left\|p-p_{h}\right\|_{0} \leq c(\alpha+h) .
$$

The natural energy norm given by

$$
\|(v, q)\|:=\left(\|v\|_{1}^{2}+\|q\|_{0}^{2}\right)^{1 / 2}
$$

will be used in the next section for the theoretical analysis of the proposed multilevel finite element VMS method.

\section{Multilevel Finite Element VMS Method}

In this section, the multilevel finite element VMS method for (16) will be presented. The stability and error estimate of it will be given. With mesh widths $h_{1}>h_{2}>\cdots>h_{f}$, the finite element space pairs $\left(X_{h_{j}}, M_{h_{j}}\right), j=1, \ldots, J$ satisfy that

$$
\left(X_{h_{1}}, M_{h_{1}}\right) \subset\left(X_{h_{2}}, M_{h_{2}}\right) \subset \cdots \subset\left(X_{h_{J}}, M_{h_{J}}\right) .
$$

And $\left(u_{h_{j}}, p_{h_{j}}\right) \in\left(X_{h_{j}}, M_{h_{j}}\right)$ is the solution obtained by the proposed multilevel finite element VMS method. The general steps of the proposed multilevel finite element VMS method for approximating the solution of (16) can be summarized as follows.
Step 1. Solve the following nonlinear system on the coarsest mesh $h_{1}$. Find $\left(u_{h_{1}}, p_{h_{1}}\right) \in\left(X_{h_{1}}, M_{h_{1}}\right)$ for all $(v, q) \in\left(X_{h_{1}}\right.$, $\left.M_{h_{1}}\right)$ satisfying

$$
\begin{aligned}
& v a\left(u_{h_{1}}, v\right)-d\left(v, p_{h_{1}}\right)+d\left(u_{h_{1}}, q\right)+G\left(u_{h_{1}}, v\right) \\
& +b\left(u_{h_{1}}, u_{h_{1}}, v\right)=(f, v) .
\end{aligned}
$$

Step 2. Update on fine mesh with Newton correction at each level. that

Give $\left(u_{h_{j-1}}, p_{h_{j-1}}\right)$ and find $\left(u_{h_{j}}, p_{h_{j}}\right) \in\left(X_{h_{j}}, M_{h_{j}}\right)$ such

$$
\begin{aligned}
& v a\left(u_{h_{j}}, v\right)-d\left(v, p_{h_{j}}\right)+d\left(u_{h_{j}}, q\right)+b\left(u_{h_{j}}, u_{h_{j-1}}, v\right) \\
& \quad+b\left(u_{h_{j-1}}, u_{h_{j}}, v\right)+G\left(u_{h_{j}}, v\right) \\
& =(f, v)+b\left(u_{h_{j-1}}, u_{h_{j-1}}, v\right)
\end{aligned}
$$

for all $(v, q) \in\left(X_{h_{j}}, M_{h_{j}}\right)$ with $j=2, \ldots, J$.

Obviously, the solution $\left(u_{h_{1}}, p_{h_{1}}\right)$ after first step of the multilevel finite element VMS method satisfies the results in Theorem 2. Before giving the stability analysis and error estimates, the continuous bilinear form $\mathscr{B}:(X, M) \times$ $(X, M) \rightarrow R$ is given by

$$
\begin{aligned}
\mathscr{B}[(u, p) ;(v, q)]:= & v a(u, v)-d(v, p)+d(u, q) \\
& +b\left(u_{h_{j}}, u, v\right)+b\left(u, u_{h_{j}}, v\right) .
\end{aligned}
$$

Here we assume that $u$ is a nonsingular solution of (1) and $u_{h_{j}}$ is close enough to $u$. Thus, by Lemma 1 and (7), the continuous bilinear form $\mathscr{B}$ satisfies the inf-sup conditions; namely, there is a constant $\gamma>0$ such that [40]

$$
\begin{array}{r}
\inf _{(u, p) \in\left(X_{h_{j}}, M_{h_{j}}\right)} \sup _{(v, q) \in\left(X_{h_{j}}, M_{h_{j}}\right)} \frac{\mathscr{B}[(u, p) ;(v, q)]}{\|(u, p)\|\|\|(v, q) \|} \geq \gamma, \\
j=1,2, \ldots, J .
\end{array}
$$

The stability analysis and error estimate of the proposed multilevel finite element method will be discussed in Theorems 3 and 4 .

Theorem 3. Suppose $X_{h_{1}} \subset X_{h_{2}} \subset X, M_{h_{1}} \subset M_{h_{2}} \subset M$, and each pair $\left(X_{h_{1}}, M_{h_{1}}\right),\left(X_{h_{2}}, M_{h_{2}}\right)$ satisfies the LBB condition (14). Let $(u, p) \in\left(H^{2}(\Omega)^{2} \cap X, H^{1}(\Omega) \cap M\right)$ be a nonsingular solution of (6). Suppose $0<h_{2}<h_{1}$ and $h_{1}$ is sufficiently small. 
Then the approximate solution $\left(u_{h_{2}}, p_{h_{2}}\right) \in\left(X_{h_{2}}, M_{h_{2}}\right)$ given by the multilevel finite element method (23) with $j=2$ satisfies

$$
\left\|\left(u_{h_{2}}, p_{h_{2}}\right)\right\| \leq k_{1,2} .
$$

Moreover, if there holds the scaled relation $h_{2}=O\left(h_{1}^{2}\right)$, it has the following error estimate:

$$
\left\|\left(u-u_{h_{2}}, p-p_{h_{2}}\right)\right\| \leq k_{2,2}\left(\alpha+h_{2}\right) .
$$

Proof. Using (5), (18), (23), (25), and (H3) gives

$$
\begin{aligned}
& \gamma\left\|\left(u_{h_{2}}, p_{h_{2}}\right)\right\| \leq \sup _{(v, q) \in\left(X^{h}, M^{h}\right)} \frac{\mathscr{B}\left[\left(u_{h_{2}}, p_{h_{2}}\right) ;(v, q)\right]}{\|(v, q)\|} \\
& \quad \leq \sup _{(v, q) \in\left(X^{h}, M^{h}\right)} \frac{\left|(f, v)+b\left(u_{h_{1}}, u_{h_{1}}, v\right)+G\left(u_{h_{2}}, v\right)\right|}{\|(v, q)\|} \\
& \leq\|f\|_{-1}+N\left\|u_{h_{1}}\right\|_{1}^{2}+C \alpha\left\|u_{h_{2}}\right\|_{1} \\
& \quad \leq\|f\|_{-1}+\frac{N}{v^{2}}\|f\|_{-1}^{2}+C \alpha\left\|\left(u_{h_{2}}, p_{h_{2}}\right)\right\| .
\end{aligned}
$$

Choosing $\alpha=O\left(h_{2}^{s}\right)$, for sufficiently small $h_{2}, \delta=\gamma-C \alpha>0$. Then we have

$$
\left\|\left(u_{h_{2}}, p_{h_{2}}\right)\right\| \leq \frac{1}{\delta}\left(\|f\|_{-1}+\frac{N}{v^{2}}\|f\|_{-1}^{2}\right) .
$$

Letting $k_{1,2}=\left(\|f\|_{-1}+\left(N / v^{2}\right)\|f\|_{-1}^{2}\right) \delta^{-1}$, then (26) holds.

Subtracting (23) with $j=2$ from (6) yields

$$
\begin{aligned}
\mathscr{B} & {\left[\left(u-u_{h_{2}}, u-p_{h_{2}}\right) ;(v, q)\right] } \\
& =b\left(u_{h_{1}}-u, u-u_{h_{1}}, v\right)+G\left(u_{h_{2}}, v\right) .
\end{aligned}
$$

The right terms in (30) are bounded according to

$$
\begin{aligned}
& \left|b\left(u_{h_{1}}-u, u-u_{h_{1}}, v\right)\right| \leq N\left\|u_{h_{1}}-u\right\|_{1}^{2}\|v\|_{1} \\
& \quad \leq c\left(\alpha+h_{1}\right)^{2}\|(v, q)\|, \\
& \left|G\left(u_{h_{2}}, v\right)\right| \leq \alpha\left\|(I-\Pi) \nabla u_{h_{2}}\right\|_{0}\|(I-\Pi) \nabla v\|_{0} \\
& \quad \leq c \alpha\left\|u_{h_{2}}\right\|_{1}\|v\|_{1} \leq c \alpha\|(v, q)\| .
\end{aligned}
$$

Taking $\left(I^{h} u, J^{h} p\right) \in\left(X_{h_{2}}, M_{h_{2}}\right)$ as interpolation of $(u, p)$ into $\left(X_{h_{2}}, M_{h_{2}}\right)$, we have

$$
\begin{aligned}
& \left\|\left(u-u_{h_{2}}, p-p_{h_{2}}\right)\right\| \leq\left\|\left(u-I^{h} u, p-J^{h} p\right)\right\| \\
& +\left\|\left(I^{h} u-u_{h_{2}}, J^{h} p-u_{h_{2}}\right)\right\| \leq c\left(\alpha+h_{2}\right)+\frac{1}{\gamma} \\
& \cdot \sup _{(v, q) \in\left(X_{h_{2}}, M_{h_{2}}\right)} \frac{\mathscr{B}\left[\left(I^{h} u-u_{h_{2}}, J^{h} p-u_{h_{2}}\right) ;(v, q)\right]}{\|(v, q)\|} \\
& \leq c\left(\alpha+h_{2}\right)+\frac{1}{\gamma} \\
& \cdot \sup _{(v, q) \in\left(X_{h_{2}}, M_{h_{2}}\right)} \frac{\left|b\left(u_{h_{1}}-u, u-u_{h_{1}}, v\right)+G\left(u_{h_{2}}, v\right)\right|}{\|(v, q)\|} \\
& \leq c\left(\alpha+h_{2}\right)+c\left(\alpha+h_{1}\right)^{2}+c \alpha .
\end{aligned}
$$

Then, together with the relation $h_{2}=O\left(h_{1}^{2}\right)$, denoting the constant as $k_{2,2}$ yields (27).

Theorem 4. Suppose $X_{h_{1}} \subset X_{h_{2}} \subset \cdots \subset X_{h_{I}} \subset X, M_{h_{1}} \subset$ $M_{h_{2}} \subset \cdots \subset M_{h_{I}} \subset M$, and each pair $\left(X_{h_{1}}, M_{h_{1}}\right), \ldots,\left(X_{h_{I}}\right.$, $\left.M_{h_{I}}\right)$ satisfies the $L B B$ condition. Let $(u, p) \in\left(H^{2}(\Omega)^{2} \cap\right.$ $\left.X, H^{1}(\Omega) \cap M\right)$ be a nonsingular solution of (6). If $h_{j}>0$ is sufficient small, then the approximate solution $\left(u_{h_{j}}, p_{h_{j}}\right) \in$ $\left(X_{h_{j}}, M_{h_{j}}\right)$ given by the multilevel finite element method (23) satisfies

$$
\left\|\left(u_{h_{j}}, p_{h_{j}}\right)\right\| \leq k_{1, j}
$$

Moreover, if there holds the scaled relation $h_{j}=O\left(h_{j-1}^{2}\right), j=$ $2,3, \ldots, J$, it has the following error estimate:

$$
\left\|\left(u-u_{h_{j}}, p-p_{h_{j}}\right)\right\|_{1} \leq k_{2, j}\left(\alpha+h_{j}\right)
$$

with $j=2, \ldots, J$.

Proof. This theorem will be proved by the induction method. From Theorem 3, it is obvious that it holds for $j=2$. Assume Theorem 4 is true for $j=m$; we need to prove that it is also true for $j=m+1$ with $1<m \leq J-1$.

Using (5), (23), (25), and (H3), we obtain

$$
\begin{aligned}
& \gamma\left\|\left(u_{h_{m+1}}, p_{h_{m+1}}\right)\right\| \mid \\
& \leq \sup _{(v, q) \in\left(X^{h}, M^{h}\right)} \frac{\mathscr{B}\left[\left(u_{h_{m+1}}, p_{h_{m+1}}\right) ;(v, q)\right]}{\|(v, q)\|} \\
& \leq \sup _{(v, q) \in\left(X^{h}, M^{h}\right)} \frac{\left|(f, v)+b\left(u_{h_{m}}, u_{h_{m}}, v\right)+G\left(u_{h_{m+1}}, v\right)\right|}{\|(v, q)\|}
\end{aligned}
$$




$$
\begin{aligned}
& \leq\|f\|_{-1}+N\left\|u_{h_{1, m}}\right\|_{1}^{2}+C \alpha\left\|u_{h_{m+1}}\right\|_{1} \\
& \leq\|f\|_{-1}+N k_{1, m}^{2}+C \alpha\left\|\left(u_{h_{m+1}}, p_{h_{m+1}}\right)\right\| .
\end{aligned}
$$

Choosing $\alpha=O\left(h_{m+1}^{s}\right)$, for sufficiently small $h_{m+1}, \delta=\gamma-$ $\mathrm{C} \alpha>0$. Then we have

$$
\left\|\left(u_{h_{m+1}}, p_{h_{m+1}}\right)\right\| \leq \frac{1}{\delta}\left(\|f\|_{-1}+N k_{1, m}^{2}\right) .
$$

Letting $k_{1, j}=\left(\|f\|_{-1}+N k_{1, m}^{2}\right) \delta^{-1}$, then (33) holds for $j=$ $m+1$.

Next, the convergence error of the multilevel finite element method is analyzed. Subtracting (23) with $j=m+1$ from (6) yields

$$
\begin{aligned}
\mathscr{B} & {\left[\left(u-u_{h_{m+1}}, u-p_{h_{m+1}}\right) ;(v, q)\right] } \\
& =b\left(u_{h_{m}}-u, u-u_{h_{m}}, v\right)+G\left(u_{h_{m+1}}, v\right) .
\end{aligned}
$$

The right terms in (37) are bounded according to

$$
\begin{aligned}
& \left|b\left(u_{h_{m}}-u, u-u_{h_{m}}, v\right)\right| \leq N\left\|u_{h_{m}}-u\right\|_{1}^{2}\|v\|_{1} \\
& \quad \leq c\left(\alpha+h_{m}\right)^{2}\|(v, q)\|, \\
& \left|G\left(u_{h_{m+1}}, v\right)\right|=\alpha\left\|(I-\Pi) \nabla u_{h_{m+1}}\right\|_{0}\|(I-\Pi) \nabla v\|_{0} \\
& \quad \leq c \alpha\left\|u_{h_{m+1}}\right\|\left\|_{1}\right\| v\left\|_{1} \leq c \alpha\right\|(v, q) \| .
\end{aligned}
$$

Taking $\left(I^{h} u, J^{h} p\right) \in\left(X_{h_{m+1}}, M_{h_{m+1}}\right)$ as interpolation of $(u, p)$ into $\left(X_{h_{m+1}}, M_{h_{m+1}}\right)$, we have

$$
\begin{aligned}
& \left\|\left(u-u_{h_{m+1}}, p-p_{h_{m+1}}\right)\right\| \leq\left\|\left(u-I^{h} u, p-J^{h} p\right)\right\| \\
& +\left\|\left(I^{h} u-u_{h_{m+1}}, J^{h} p-u_{h_{m+1}}\right)\right\| \leq c\left(\alpha+h_{m+1}\right)+\frac{1}{\gamma} \\
& \cdot \sup _{(v, q) \in\left(X_{h_{m+1}}, M_{h_{m+1}}\right)} \frac{\mathscr{B}\left[\left(I^{h} u-u_{h_{m+1}}, J^{h} p-u_{h_{m+1}}\right) ;(v, q)\right]}{\|(v, q)\| \mid} \\
& \leq c\left(\alpha+h_{m+1}\right)+\frac{1}{\gamma} \\
& \quad \sup _{(v, q) \in\left(X_{h_{m+1}}, M_{h_{m+1}}\right)} \frac{\left|b\left(u_{h_{m}}-u, u-u_{h_{m}}, v\right)+G\left(u_{h_{m+1}}, v\right)\right|}{\|(v, q)\|} \\
& \leq c\left(\alpha+h_{m+1}\right)+c\left(\alpha+h_{m}\right)^{2}+c \alpha .
\end{aligned}
$$

Then, together with the relation $h_{m+1}=O\left(h_{m}^{2}\right)$, denoting the constant as $k_{2, m+1}$ yields (34) for $j=m+1$. Thus, Theorem 4 follows from the induction principle.

\section{Numerical Examples}

In this section, three numerical examples are given: the first one is an exact solution problem, the second one is the lid-driven cavity problem, and the third one is the backward facing step problem. The mentioned methods in this paper are all implemented using Freefem ++ [43] and the convergence tolerance is set equal to $10^{-6}$. In the numerical examples, the triangular mesh with the Taylor-Hood element pair is used for the finite element discretization. For the sake of simplicity, the one-level and the proposed multilevel finite element VMS methods are written as $j$-level finite element VMS method in the numerical examples. The nonlinear systems in these methods are all linearized by the Oseen iterative method (see $[44,45]$ ).

5.1. Problem with an Exact Solution. This problem is a steady flow of incompressible viscous Newtonian fluid in bounded domain $\Omega=(0,1) \times(0,1)$ with $u=(0,0)$ on the boundaries. It has the following exact solution:

$$
\begin{aligned}
& u_{1}=10 x^{2}(x-1)^{2} y(y-1)(2 y-1), \\
& u_{2}=-10 x(x-1)(2 x-1) y^{2}(y-1)^{2}, \\
& p=10(2 x-1)(2 y-1),
\end{aligned}
$$

where $f=\left(f_{1}, f_{2}\right)$ is determined by (1). The Taylor-Hood elements predict a convergence rate of $O\left(h^{2}\right)$ in the energy norm (i.e., $H^{1}$-norm for the velocity and $L^{2}$-norm for the pressure), so the eddy viscosity parameter is chosen as $\alpha=$ $0.1 h_{j}^{2}$ for multilevel finite element VMS method which is the same as that used in [21].

First, $v=0.1$ is considered. The meshes for the one-level finite element VMS method are chosen as $h_{J}=\left[1 / 8^{2} ; 1 / 10^{2}\right.$; $\left.1 / 12^{2} ; 1 / 14^{2} ; 1 / 16^{2}\right]$, and these meshes are also the finest meshes for the multilevel finite element VMS method. The choices for the coarse meshes of the multilevel finite element VMS method are very important. If the coarse meshes are not fine enough, the optimal convergence orders of velocity and pressure can not be reached. According to Theorems 3 and 4 , the coarse meshes $h_{j-1}$ and the fine mesh $h_{j}$ should be chosen as $h_{j} \sim h_{j-1}^{2}, j=2,3, \ldots, J$, which ensure optimal accuracy of the approximate solution. Here we consider the multilevel finite element VMS method for $j=2,3,4$, and meshes are chosen as $h_{j}=h_{j-1}^{2}$, respectively. The numerical results using the one-level and the proposed multilevel finite element VMS methods are shown in Figure 1, which indicates that the proposed multilevel finite element VMS method can reach the convergence order of $\mathrm{O}\left(h^{2}\right)$ for velocity in $H^{1}$-norm and pressure in $L^{2}$-norm and the optimal convergence order of $\mathrm{O}\left(h^{3}\right)$ for velocity in the $L^{2}$-norm. These numerical results are in agreement with the theoretical predictions. Moreover, 
TABLE 1: Comparison of these $j$-level finite element VMS methods.

\begin{tabular}{lccc||cc||c}
\hline Method & CPU(s) & $\frac{\left\|u-u_{h_{j}}\right\|_{0}}{\|u\|_{0}}$ & $\frac{\left\|u-u_{h_{j}}\right\|_{1}}{\|u\|_{1}}$ & $\frac{\left\|p-p_{h_{j}}\right\|_{0}}{\|p\|_{0}}$ \\
\hline 1-level & $1 / h$ & 198.614 & $1.37319 e-005$ & 0.000776894 & $4.72788 e-005$ \\
2-level & 128 & 71.389 & $1.02514 e-005$ & 0.000351524 & $4.72788 e-005$ \\
3-level & $64-128$ & 37.617 & $2.11498 e-005$ & 0.000342189 & $4.72788 e-005$ \\
4-level & $32-64-128$ & 29.495 & 0.000110742 & 0.000526664 & $4.7279 e-005$ \\
\hline 1-level & $16-32-64-128$ & 250.86 & $8.5448 e-006$ & 0.000554574 & $3.73553 e-005$ \\
2-level & 144 & 92.251 & $5.50229 e-006$ & 0.000226809 & $3.73553 e-005$ \\
3-level & $72-144$ & 45.212 & $9.30361 e-006$ & 0.000206441 & $3.73553 e-005$ \\
4-level & $36-72-144$ & 35.437 & $4.3288 e-005$ & 0.000275735 & $3.73553 e-005$ \\
\hline 1-level & $18-36-72-144$ & 925.036 & $9.02168 e-007$ & 0.000107772 & $1.18393 e-005$ \\
2-level & 256 & 307.479 & $2.85225 e-007$ & $4.66203 e-005$ & $1.18403 e-005$ \\
3-level & $128-256$ & 141.673 & $2.02856 e-007$ & $4.5437 e-005$ & $1.18383 e-005$ \\
4-level & $64-128-256$ & 99.477 & $1.46991 e-007$ & $4.50338 e-005$ & $1.18391 e-005$ \\
\hline
\end{tabular}

both the proposed multilevel finite element VMS method and the one-level finite element VMS method obtain nearly the same approximation results, but the former significantly saves computing time compared with the later.

Then $v=0.0001$ is considered. The mesh, CPU-time, the $L^{2}$-error of the velocity, $H^{1}$-error of the velocity, and $L^{2}$-error of the pressure for comparison of these $j$-level $(j=1,2,3,4)$ finite element VMS methods are tabulated in Table 1. Here $\left(u_{h_{j}}, p_{h_{j}}\right)$ refers to the approximation solution obtained by $j$ level finite element VMS method. Table 1 indicates that $j$-level $(j \geq 2)$ finite element VMS method saves much computing time compared with $j-1$-level finite element VMS method at the same fine mesh, and the finer the mesh becomes, the smaller the error achieves. Furthermore, when scrutinizing the error estimates of velocity and the pressure in Table 1, it is easily observed that multilevel $(j=2,3,4)$ finite element VMS method for the velocity and the pressure is better than one-level $(j=1)$ finite element VMS method.

5.2. Lid-Driven Cavity Problem. Lid-driven cavity problem often serves as a standard benchmark problem for incompressible Navier-Stokes equations because the benchmark data is available for comparison. In this problem, the computation is carried out in the region $\Omega=\{(x, y) \mid 0<x, y<1\}$. The velocity is $u=(1,0)$ on the top side $\{(x, 1): 0<x<1\}$ and $u=(0,0)$ on the rest of the boundary.

We compute the approximate solutions for $\mathrm{Re}=$ $(1000 ; 5000 ; 10000)$ and then compare the results of 1-level finite element VMS method $(1 / h=256)$, 2-level finite element VMS method $\left(1 / h_{1}=128,1 / h_{2}=256\right)$, 3-level finite element VMS method $\left(1 / h_{1}=64,1 / h_{2}=128,1 / h_{3}=256\right)$, and 4-level finite element VMS method $\left(1 / h_{1}=64,1 / h_{2}=\right.$ $\left.64,1 / h_{3}=128,1 / h_{4}=256\right)$ with that of Ghia et al. [1]. Figures 2-4 plot the horizontal component of the velocity along the vertical line passing through the geometrical center of the cavity and the vertical component of the velocity along the horizontal line passing through the geometrical center of the cavity, respectively. As shown in Figure 2, the results of multilevel finite element VMS method are
TABle 2: Computing time of these $j$-level finite element VMS methods.

\begin{tabular}{lcccc}
\hline Re & 1-level & 2-level & 3-level & 4-level \\
\hline 1000 & 1625.09 & 501.335 & 193.591 & 115.911 \\
5000 & 2986.47 & 888.397 & 452.884 & 347.089 \\
10000 & 4399.63 & 1560.04 & 670.384 & 322.642 \\
\hline
\end{tabular}

indistinguishable from 1-level finite element VMS method and the benchmark data in [1]. However, in Figures 3 and 4, the 4-level finite element VMS method at $\mathrm{Re}=5000$ and the 3-level and 4-level finite element VMS method at $\mathrm{Re}=10000$ provide unsatisfactory results. This is because the nonlinear solution on the initial coarse mesh is not accurate enough. Actually, with an appropriate choice of initial coarse mesh, the multilevel finite element VMS method can get better results than one-level finite element VMS method. Table 2 presents the computing time of these $j$-level $(j=1,2,3,4)$ finite element VMS methods and demonstrates that $j$-level finite element VMS method saves much computing time compared with $j$-1-level $(j=2,3,4)$ finite element VMS method.

5.3. Backward Facing Step Problem. Backward facing step problem is also a standard numerical example which involves a steady viscous incompressible flow over an isothermal two-dimensional backward facing step. It is well-known that the backward facing step problem possesses a corner singularity; thus it is often adopted by many researchers to verify their numerical methods. The geometry and the boundary conditions are shown in Figure 5 as in $[21,46]$.

In this example, the Reynolds number is 150 which is based on the maximum inlet velocity $v_{x}(\max )=1$ and the height of the inlet. We compute an approximate solution on the following number of triangle elements: 9344 (for 1-level finite element VMS method), 5298-9344 (for 2-level finite element VMS method), 2392-5298-9344 (for 3-level finite element VMS method), and 646-2392-5298-9344 (for 4-level finite element VMS method). Figures 6(a)-6(d) show the 


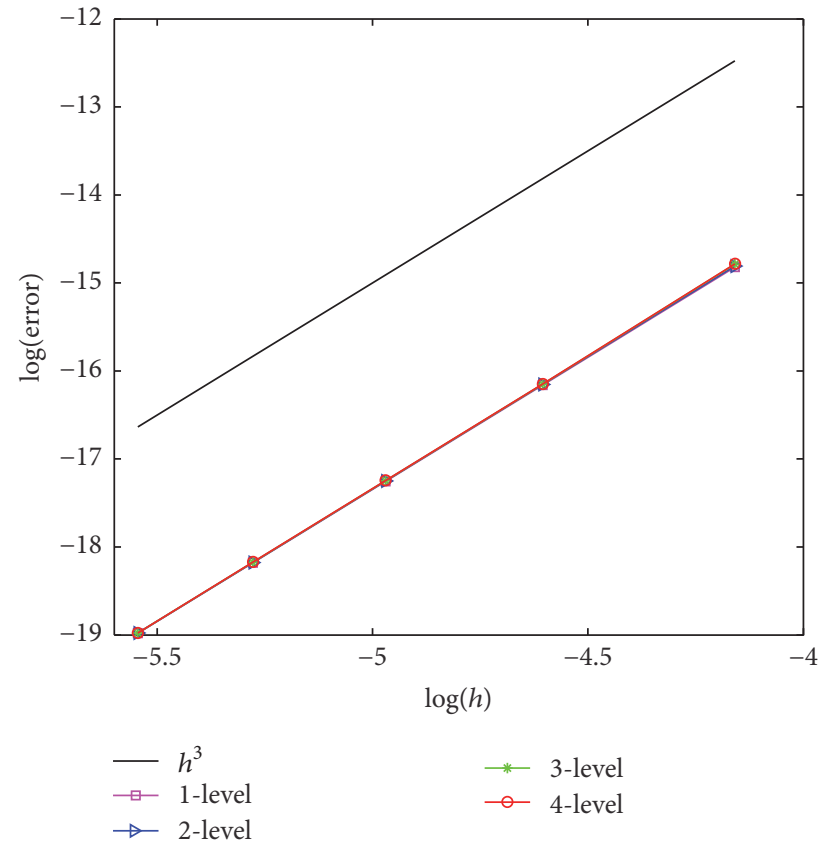

(a)

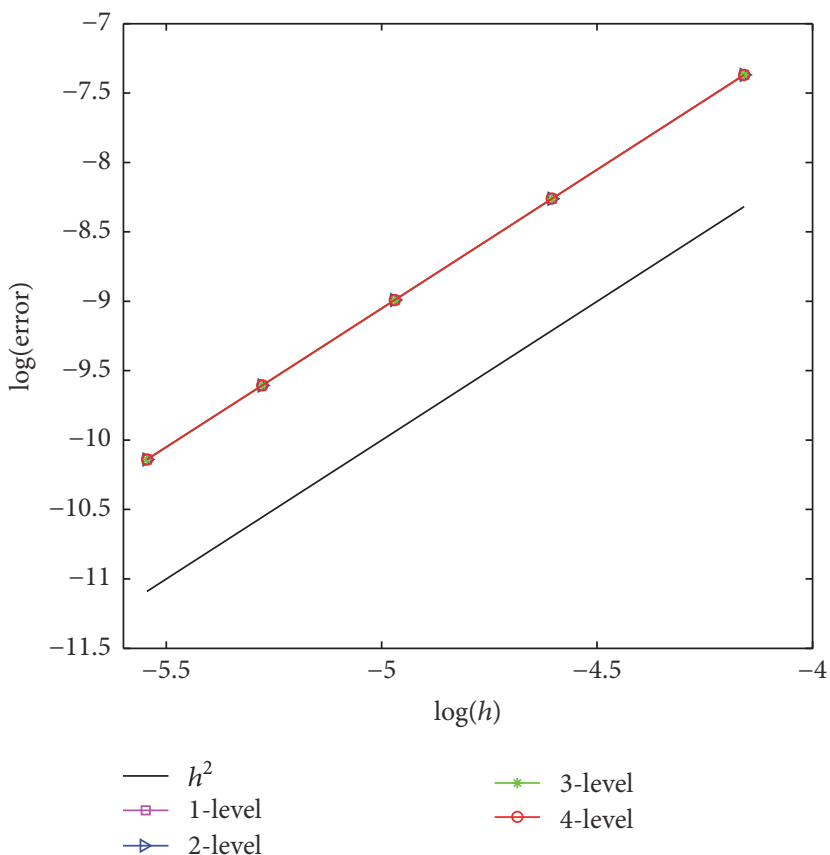

(c)

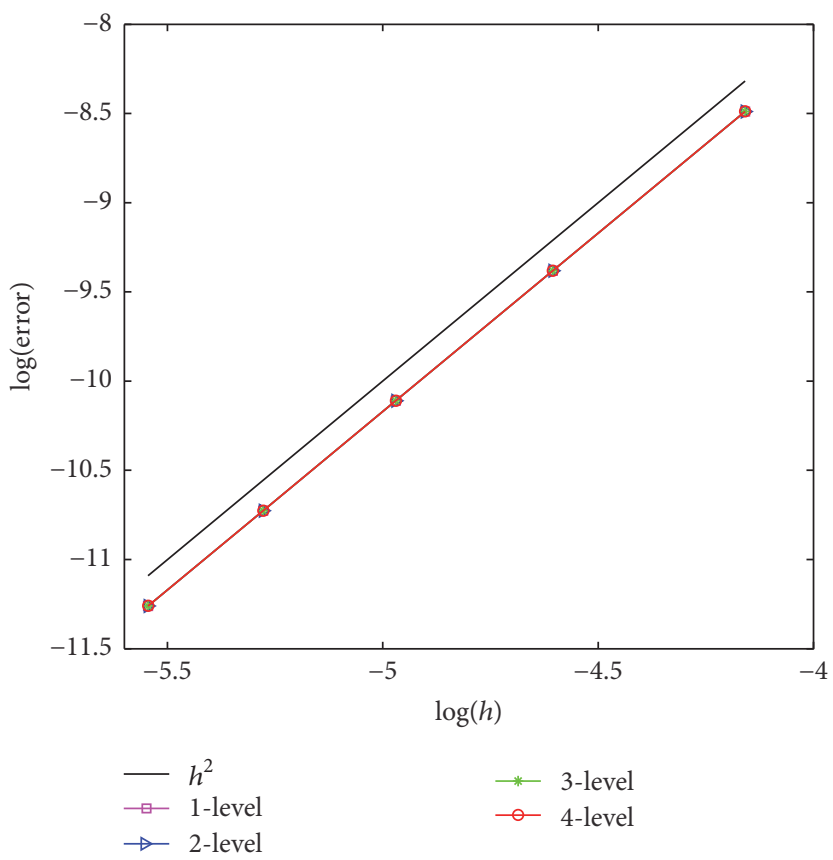

(b)

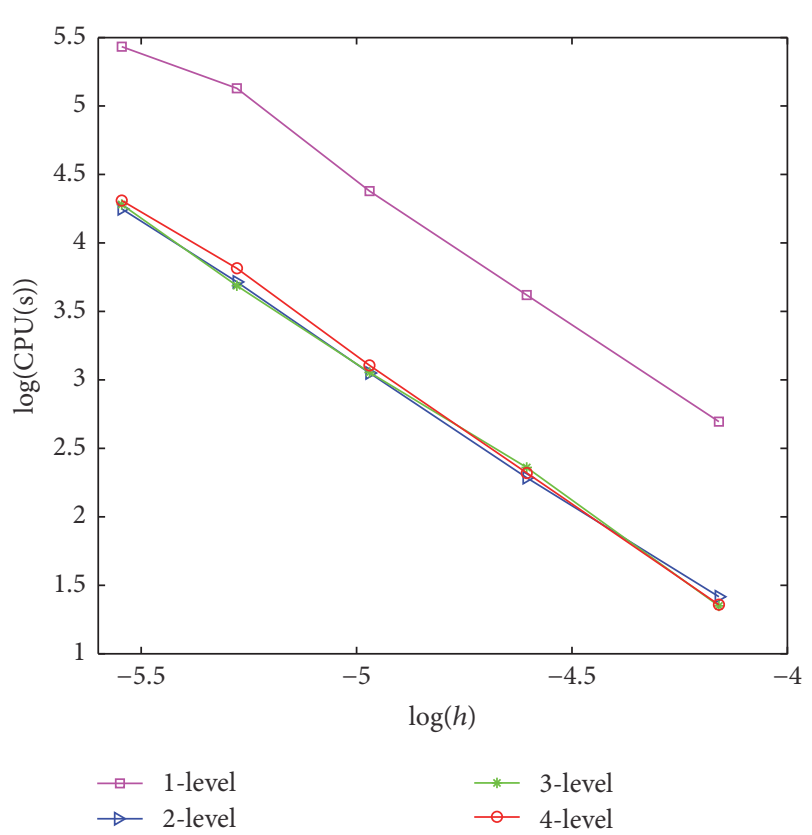

(d)

FIGURE 1: Convergence analysis for the velocity and the pressure using $j$-level $(j=1,2,3,4)$ finite element VMS method. (a) $L^{2}$ error for the velocity; (b) $H^{1}$ error for the velocity; (c) $L^{2}$ error for the pressure; (d) computing time.

pressure field and the streamlines obtained by these $j$-level $(j=1,2,3,4)$ finite element VMS methods, respectively, and these results are in agreement with those of $[21,46]$. In order to observe these results more clearly, a comparison of the computed velocity, pressure, and vorticity is given across the channel at $x=1.5$ in Figures $7(\mathrm{a})-7(\mathrm{~d})$, it is obvious that the results of these $j$-level $(j=1,2,3,4)$ finite element
VMS methods nearly have no differences with each other in velocity and vorticity, but the pressure computed by 4 level finite element VMS method has a clear difference from others. The reason is that the initial grid chosen as 646 in 4-level finite element VMS method is too coarse. Table 3 presents the computing time of these $j$-level $(j=1,2,3,4)$ finite element VMS methods; it shows that the $j$-level finite 


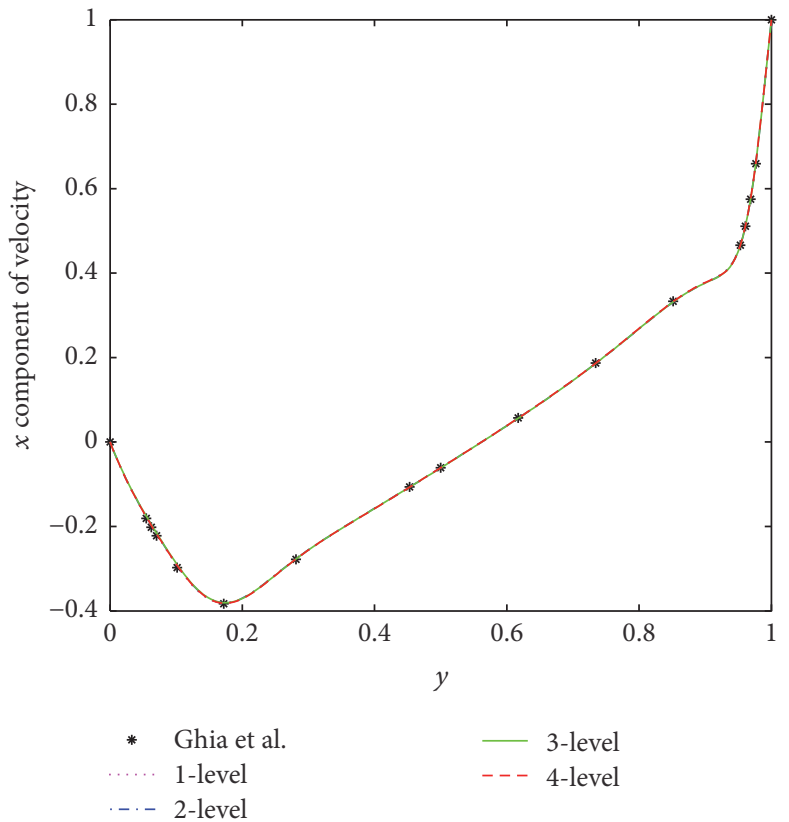

(a)

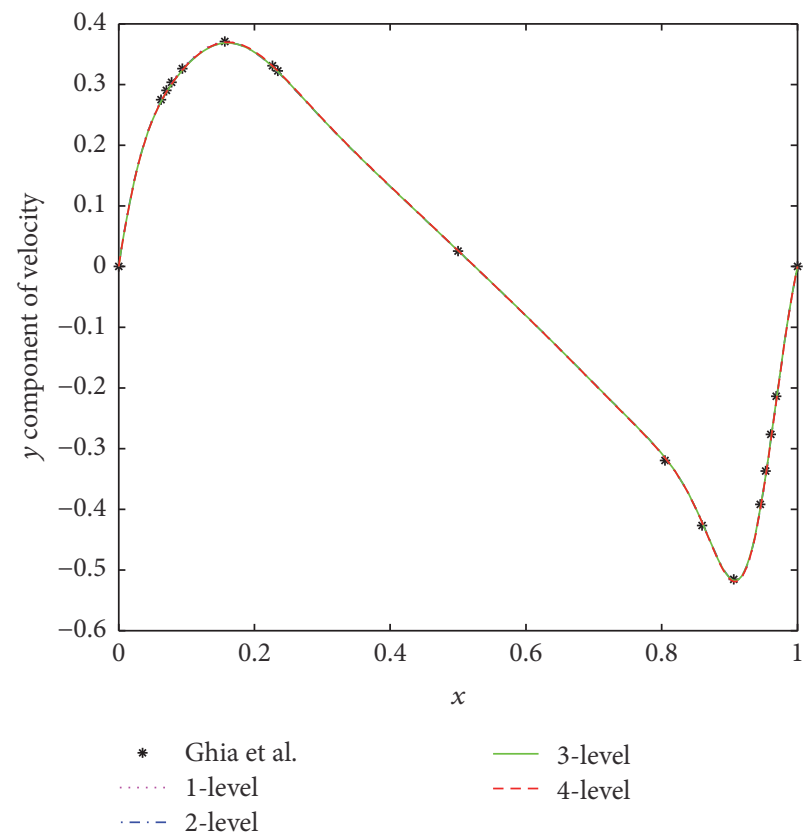

(b)

FIGURE 2: Horizontal (a) and vertical (b) of velocity by $j$-level finite element VMS method at $\mathrm{Re}=1000$.

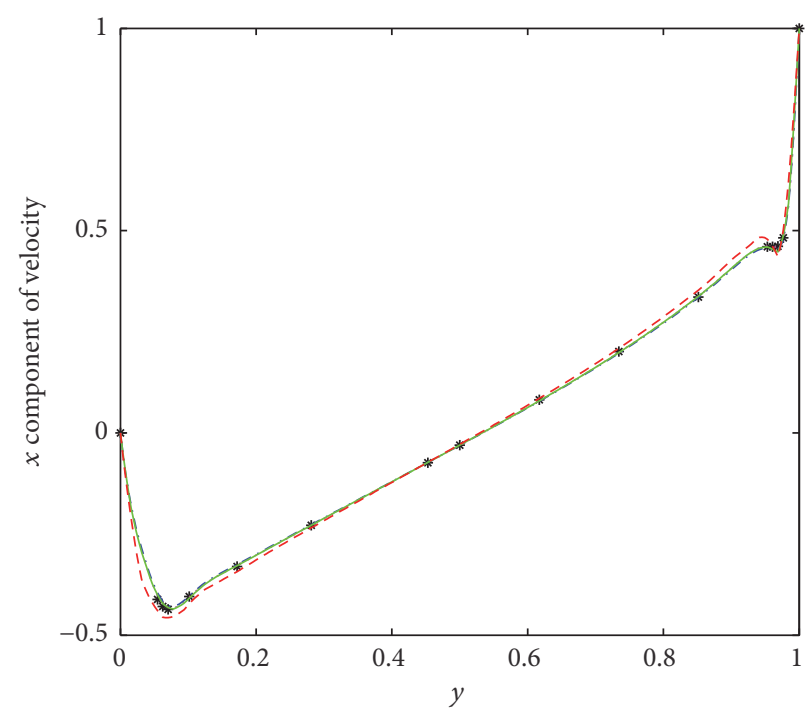

* Ghia et al. 1-level -.. 2-level

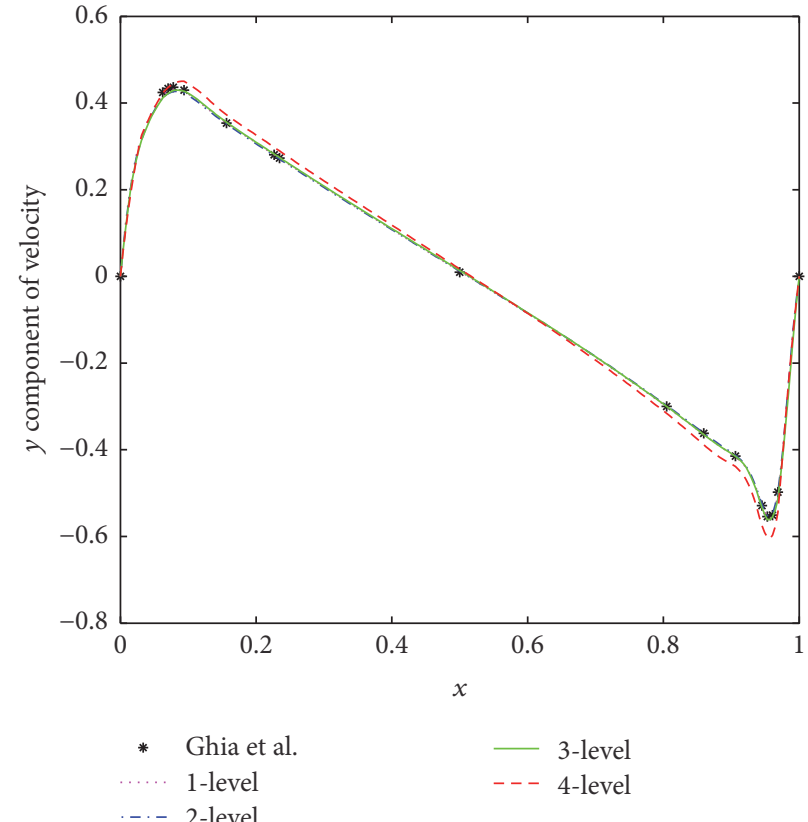

(b)

FIgURE 3: Horizontal (a) and vertical (b) of velocity by $j$-level finite element VMS method at $\operatorname{Re}=5000$.

element VMS method saves much computing time compared with $j-1$-level finite element VMS method. From this example, it can be concluded that the proposed multilevel finite element VMS method is also efficient for problems with a corner singularity, and it is a highly effective and timesaving method if the coarse grid is chosen appropriately.

\section{Conclusions}

In this paper, a multilevel finite element variational multiscale method based on two local Gauss integrations is proposed and applied to the numerical simulation of steady incompressible Navier-Stokes equations. Theoretically, the stability 


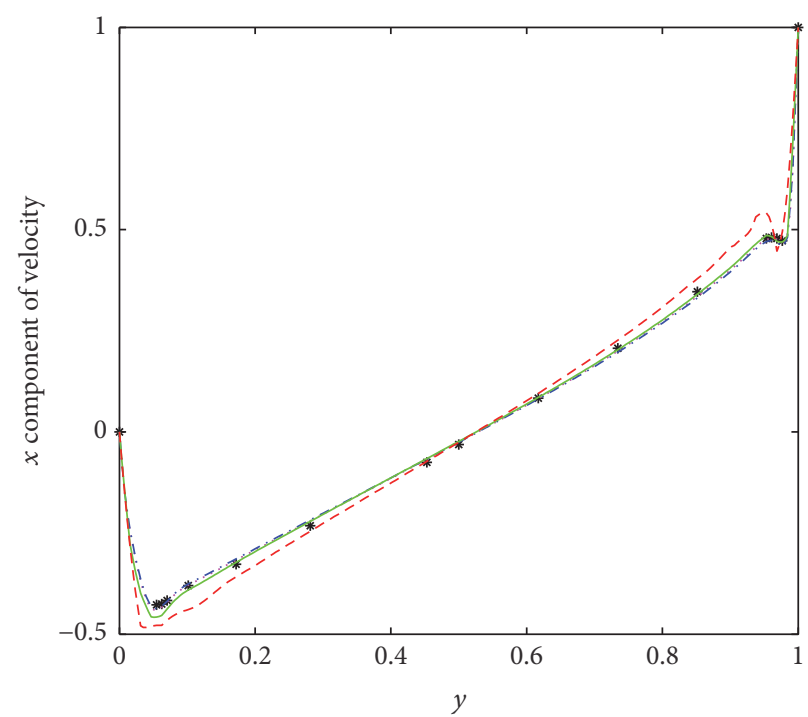

* Ghia et al.

1-level

...- 2-level

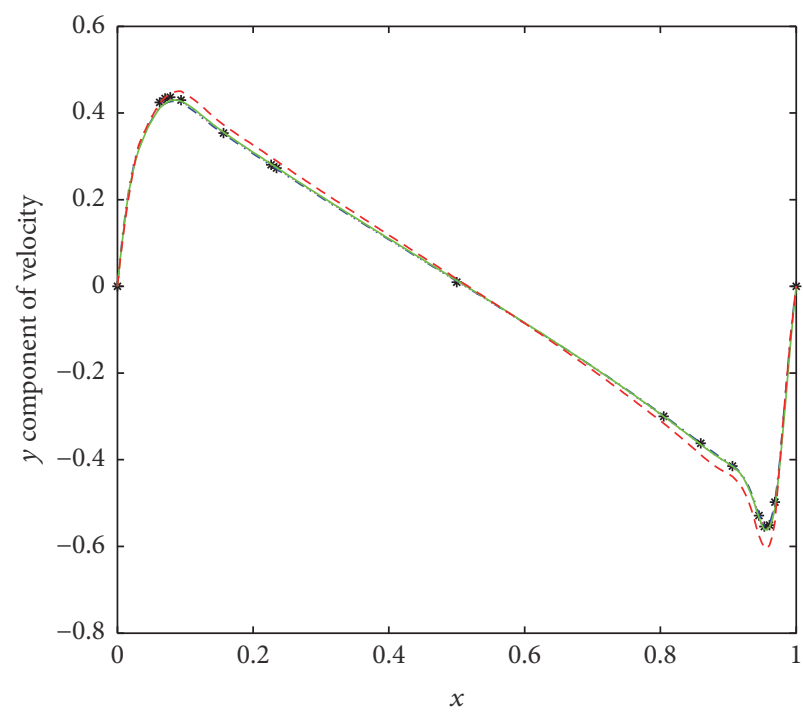

* Ghia et al.

1-level

-..- 2-level

(a)

(b)

FIGURE 4: Horizontal (a) and vertical (b) of velocity by $j$-level finite element VMS method at Re $=10000$.

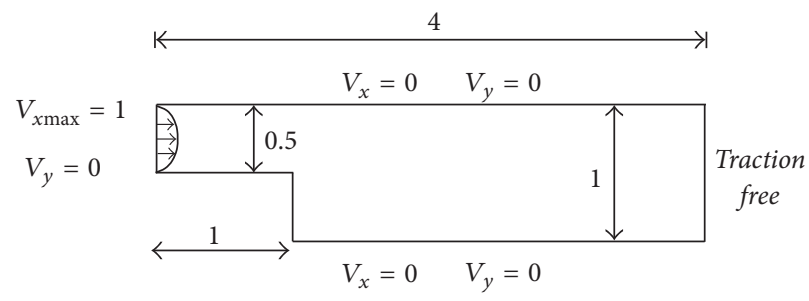

FIGURE 5: Problem description of the backward facing step.

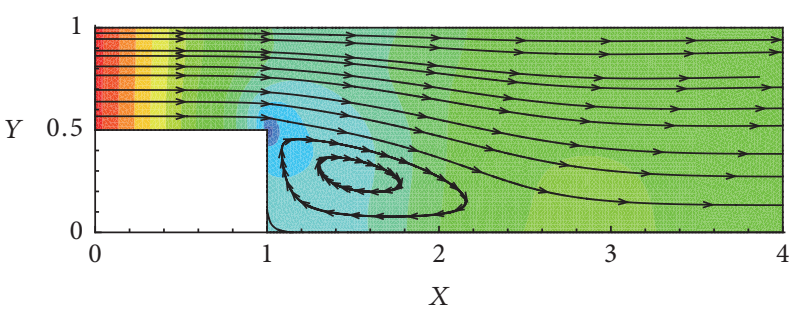

(a) 1-level finite element VMS method

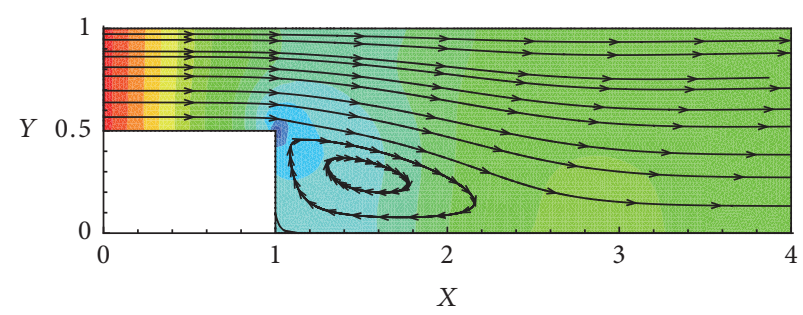

(c) 3-level finite element VMS method

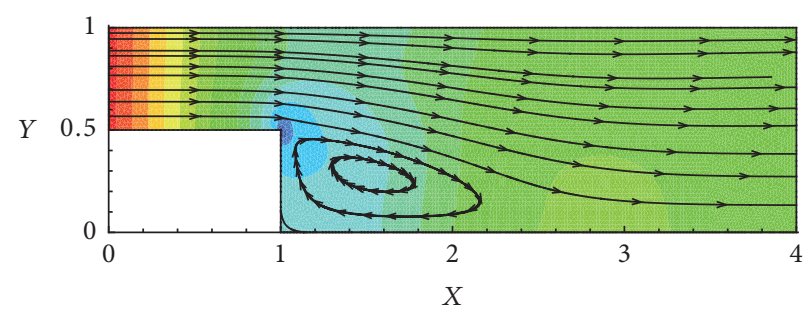

(b) 2-level finite element VMS method

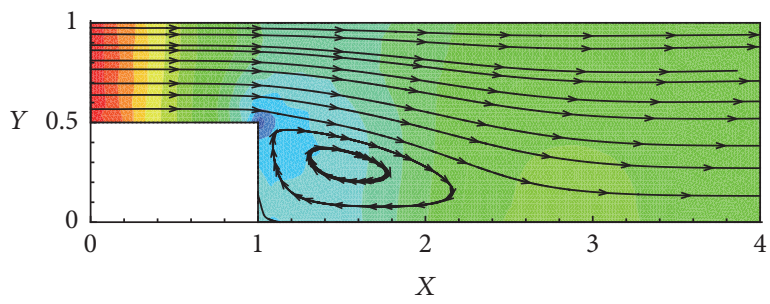

(d) 4-level finite element VMS method

Figure 6: Computed pressure field and streamlines by these $j$-level $(j=1,2,3,4)$ finite element VMS methods. 


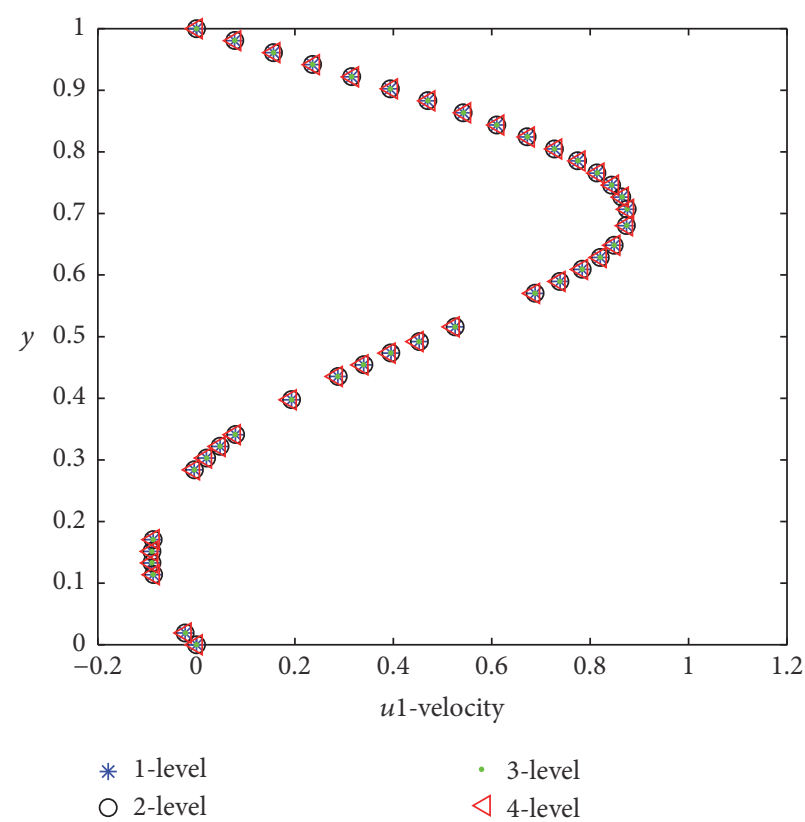

(a) $u_{1}$-velocity at $x=1.5$

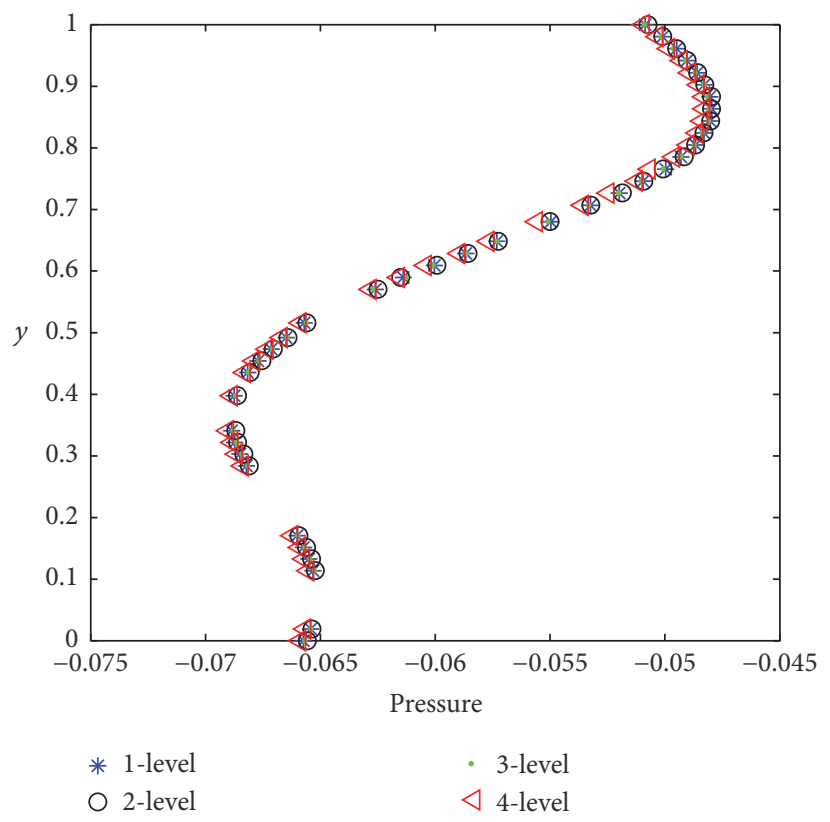

(c) Pressure at $x=1.5$

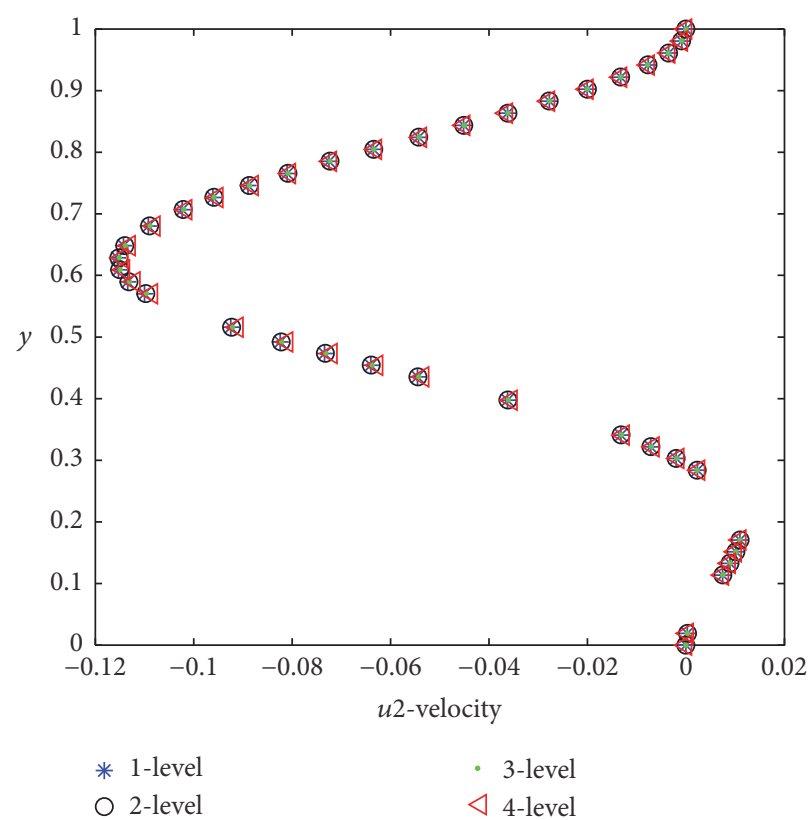

(b) $u_{2}$-velocity at $x=1.5$

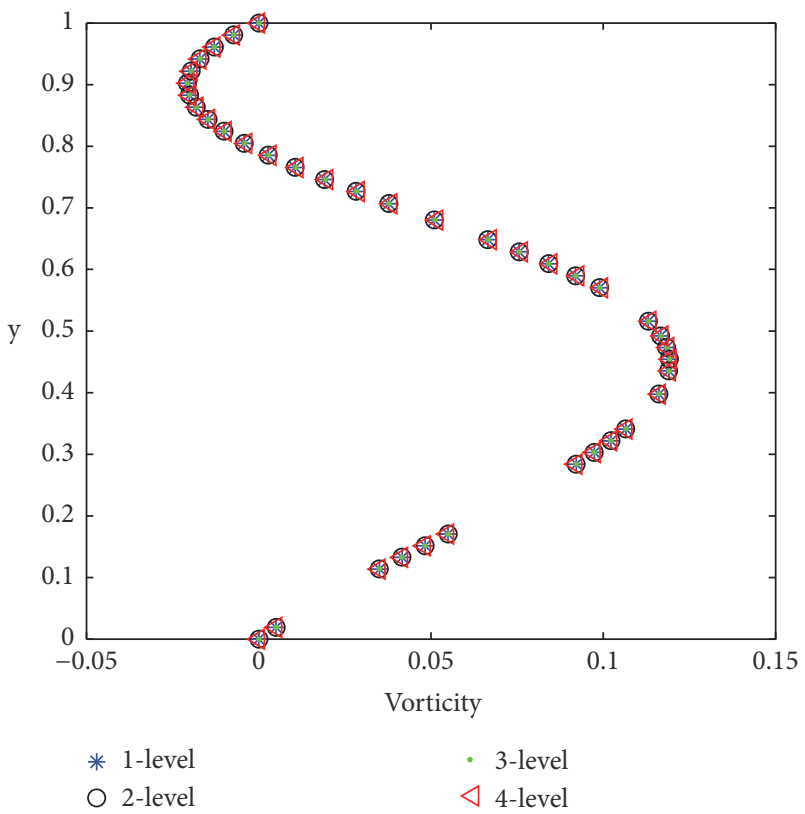

(d) Vorticity at $x=1.5$

FIGURE 7: Comparison of $u_{1}$-velocity, $u_{2}$-velocity, pressure, and vorticity profiles at $x=1.5$.

TABLE 3: Computing time of these $j$-level finite element VMS methods.

\begin{tabular}{lcccc}
\hline Method & 1-level & 2-level & 3-level & 4-level \\
\hline Computing time & 157.896 & 98.153 & 56.247 & 31.261 \\
\hline
\end{tabular}

and error estimate of the multilevel finite element variational multiscale method are analyzed. Numerically, three numerical examples are given. The numerical results demonstrate that, under an appropriate initial coarse mesh, the multilevel finite element variational multiscale method is more efficient than standard one-level finite element variational multiscale method. In addition, this multilevel finite element variational multiscale method can be extended to solve other more complex fluid dynamical models which will be discussed in further work.

\section{Conflicts of Interest}

The authors declare that there are no conflicts of interest regarding the publication of this article. 


\section{Acknowledgments}

The project is supported by 973 Program (no. 2012CB026002), the NSF of China (no. 51305355), and the National Key Technology R\&D Program of China (no. 2013BAF01B02).

\section{References}

[1] U. Ghia, K. N. Ghia, and C. T. Shin, "High-Re solutions for incompressible flow using the Navier-Stokes equations and a multigrid method," Journal of Computational Physics, vol. 48, no. 3, pp. 387-411, 1982.

[2] E. Erturk, T. C. Corke, and C. Gökçöl, "Numerical solutions of 2-D steady incompressible driven cavity flow at high Reynolds numbers," International Journal for Numerical Methods in Fluids, vol. 48, no. 7, pp. 747-774, 2005.

[3] Y. Shang, "A two-level subgrid stabilized Oseen iterative method for the steady Navier-Stokes equations," Journal of Computational Physics, vol. 233, pp. 210-226, 2013.

[4] L. P. Franca and S. L. Frey, "Stabilized finite element methods. II. The incompressible Navier-Stokes equations," Computer Methods in Applied Mechanics and Engineering, vol. 99, no. 23, pp. 209-233, 1992.

[5] L. P. Franca and T. J. Hughes, "Convergence analyses of Galerkin least-squares methods for symmetric advective-diffusive forms of the STOkes and incompressible Navier-STOkes equations," Computer Methods in Applied Mechanics and Engineering, vol. 105, no. 2, pp. 285-298, 1993.

[6] A. N. Brooks and T. J. Hughes, "Streamline upwind/PetrovGalerkin formulations for convection dominated flows with particular emphasis on the incompressible Navier-Stokes equations," Computer Methods in Applied Mechanics and Engineering, vol. 32, no. 1-3, pp. 199-259, 1982.

[7] W. Layton, H. K. Lee, and J. Peterson, "A defect-correction method for the incompressible Navier-Stokes equations," Applied Mathematics and Computation, vol. 129, no. 1, pp. 1-19, 2002.

[8] Z. Si and Y. He, "A defect-correction mixed finite element method for stationary conduction-convection problems," Mathematical Problems in Engineering, vol. 2011, Article ID 370192, 28 pages, 2011.

[9] D. Arndt, H. Dallmann, and G. Lube, "Local projection FEM stabilization for the time-dependent incompressible NavierStokes problem," Numerical Methods for Partial Differential Equations. An International Journal, vol. 31, no. 4, pp. 1224-1250, 2015.

[10] A. Montlaur, S. Fernandez-Mendez, J. Peraire, and A. Huerta, "Discontinuous Galerkin methods for the Navier-Stokes equations using solenoidal approximations," International Journal for Numerical Methods in Fluids, vol. 64, no. 5, pp. 549-564, 2010.

[11] N. C. Nguyen, J. Peraire, and B. Cockburn, "An implicit highorder hybridizable discontinuous Galerkin method for the incompressible Navier-Stokes equations," Journal of Computational Physics, vol. 230, no. 4, pp. 1147-1170, 2011.

[12] B. Riviere and S. Sardar, "Penalty-free discontinuous Galerkin methods for incompressible Navier-Stokes equations," Mathematical Models and Methods in Applied Sciences, vol. 24, no. 6, pp. 1217-1236, 2014.

[13] T. J. R. Hughes, L. Mazzei, and K. E. Jansen, "Large Eddy Simulation and the variational multiscale method," Computing and Visualization in Science, vol. 3, no. 1-2, pp. 47-59, 2000.
[14] T. J. R. Hughes, L. Mazzei, A. A. Oberai, and A. A. Wray, "The multiscale formulation of large eddy simulation: Decay of homogeneous isotropic turbulence," Physics of Fluids, vol. 13, no. 2, pp. 505-512, 2001.

[15] T. J. R. Hughes, A. A. Oberai, and L. Mazzei, "Large eddy simulation of turbulent channel flows by the variational multiscale method," Physics of Fluids, vol. 13, no. 6, pp. 1784-1799, 2001.

[16] T. J. R. Hughes, V. M. Calo, and G. Scovazzi, Variational and Multiscale Methods in Turbulence, Mechanics of the 21st Century, 2005, 153-163.

[17] J.-L. Guermond, "Stabilization of Galerkin approximations of transport equations by subgrid modeling," M2AN. Mathematical Modelling and Numerical Analysis, vol. 33, no. 6, pp. 12931316, 1999.

[18] W. Layton, "A connection between subgrid scale eddy viscosity and mixed methods," Applied Mathematics and Computation, vol. 133, no. 1, pp. 147-157, 2002.

[19] V. John and S. Kaya, "A finite element variational multiscale method for the Navier-Stokes equations," SIAM Journal on Scientific Computing, vol. 26, no. 5, pp. 1485-1503, 2005.

[20] S. S. Collis, "Monitoring unresolved scales in multiscale turbulence modeling," Physics of Fluids, vol. 13, no. 6, pp. 1800-1806, 2001.

[21] H. Zheng, Y. Hou, F. Shi, and L. Song, "A finite element variational multiscale method for incompressible flows based on two local Gauss integrations," Journal of Computational Physics, vol. 228, no. 16, pp. 5961-5977, 2009.

[22] F. Shi, H. Zheng, J. Yu, and Y. Li, "On the convergence of variational multiscale methods based on Newton's iteration for the incompressible flows," Applied Mathematical Modelling. Simulation and Computation for Engineering and Environmental Systems, vol. 38, no. 23, pp. 5726-5742, 2014.

[23] J. Yu, H. Zheng, and F. Shi, "A finite element variational multiscale method for incompressible flows based on the construction of the projection basis functions," International Journal for Numerical Methods in Fluids, vol. 70, no. 6, pp. 793804, 2012.

[24] Y. Shang and S. Huang, "A parallel subgrid stabilized finite element method based on two-grid discretization for simulation of 2D/3D Steady incompressible flows," Journal of Scientific Computing, vol. 60, no. 3, pp. 564-583, 2014.

[25] Y. Jiang, L. Mei, H. Wei, W. Tian, and J. Ge, "A finite element variational multiscale method based on two local gauss integrations for stationary conduction-convection problems," Mathematical Problems in Engineering, vol. 2012, Article ID 747391, 14 pages, 2012.

[26] W. Layton and H. W. Lenferink, "A multilevel mesh independence principle for the Navier-Stokes equations," SIAM Journal on Numerical Analysis, vol. 33, no. 1, pp. 17-30, 1996.

[27] C. Calgaro, A. Debussche, and J. Laminie, "On a multilevel approach for the two-dimensional Navier-Stokes equations with finite elements," International Journal for Numerical Methods in Fluids, vol. 27, no. 1-4, Special Issue, pp. 241-258, 1998.

[28] W. Layton, H. K. Lee, and J. Peterson, "Numerical solution of the stationary Navier-Stokes equations using a multilevel finite element method," SIAM Journal on Scientific Computing, vol. 20, no. 1, pp. 1-12, 1998.

[29] Y. He and K.-M. Liu, "A multilevel finite element method in space-time for the Navier-Stokes problem," Numerical Methods for Partial Differential Equations. An International Journal, vol. 21, no. 6, pp. 1052-1078, 2005. 
[30] J. Li, Y. He, and H. Xu, "A multi-level stabilized finite element method for the stationary Navier-Stokes equations," Computer Methods in Applied Mechanics and Engineering, vol. 196, no. 2930, pp. 2852-2862, 2007.

[31] J. Li, Z. Chen, and Y. He, "A stabilized multi-level method for non-singular finite volume solutions of the stationary $3 \mathrm{D}$ Navier-Stokes equations," Numerische Mathematik, vol. 122, no. 2, pp. 279-304, 2012.

[32] X. Zhao, J. Li, J. Su, and G. Lei, "Analysis of newton multilevel stabilized finite volume method for the three-dimensional stationary Navier-Stokes equations," Numerical Methods for Partial Differential Equations, vol. 29, no. 6, pp. 2146-2160, 2013.

[33] P. Kopp, Multi-level hp-FEM and the Finite Cell Method for the Navier-Stokes equations using a Variational Multiscale Formulation [M.S. thesis], Technische Universitat Munchen, 2017.

[34] V. John, S. Kaya, and W. Layton, "A two-level variational multiscale method for convection-dominated convection-diffusion equations," Computer Methods in Applied Mechanics and Engineering, vol. 195, no. 33-36, pp. 4594-4603, 2006.

[35] Y. Li, L. Mei, Y. Li, and K. Zhao, "A two-level variational multiscale method for incompressible flows based on two local Gauss integrations," Numerical Methods for Partial Differential Equations, vol. 29, no. 6, pp. 1986-2003, 2013.

[36] R. Temam, Navier-Stokes Equations, North-Holland, Amsterdam, The Netherlands, 1984.

[37] S. Kaya, W. Layton, and B. Rivire, "Subgrid stabilized defect correction methods for the Navier-Stokes equations," SIAM Journal on Numerical Analysis, vol. 44, no. 4, pp. 1639-1654, 2006.

[38] Y. He, "Stability and convergence of iterative methods related to viscosities for the 2D/3D Steady Navier-Stokes equations," Journal of Mathematical Analysis and Applications, vol. 423, no. 2, pp. 1129-1149, 2015.

[39] F. Brezzi and M. Fortin, Mixed and Hybrid Finite Element Methods, Springer, Berlin, Germany, 1991.

[40] W. Layton and L. Tobiska, "A two-level method with backtracking for the Navier-Stokes equations," SIAM Journal on Numerical Analysis, vol. 35, no. 5, pp. 2035-2054, 1998.

[41] C. Taylor and P. Hood, "A numerical solution of the NavierStokes equations using the finite element technique," Computers and Fluids, vol. 1, no. 1, pp. 73-100, 1973.

[42] V. John and S. Kaya, "Finite element error analysis of a variational multiscale method for the Navier-Stokes equations," Advances in Computational Mathematics, vol. 28, no. 1, pp. 4361, 2008.

[43] “Freefem++," version 3.38, http://www.freefem.org/.

[44] Y. He and J. Li, "Convergence of three iterative methods based on the finite element discretization for the stationary NavierStokes equations," Computer Methods in Applied Mechanics and Engineering, vol. 198, no. 15-16, pp. 1351-1359, 2009.

[45] R. An, "Comparisons of STOkes/Oseen/Newton iteration methods for NAVier-STOkes equations with friction boundary conditions," Applied Mathematical Modelling. Simulation and Computation for Engineering and Environmental Systems, vol. 38, no. 23, pp. 5535-5544, 2014.

[46] A. Masud and R. A. Khurram, "A multiscale finite element method for the incompressible Navier-Stokes equations," Computer Methods in Applied Mechanics and Engineering, vol. 195, no. 13-16, pp. 1750-1777, 2006. 


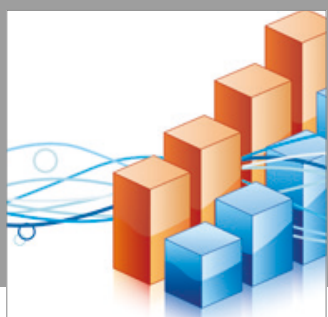

Advances in

Operations Research

vatersals

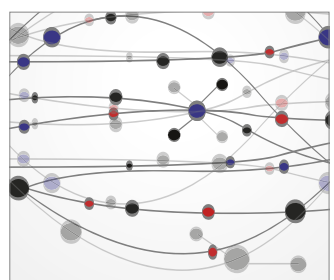

\section{The Scientific} World Journal
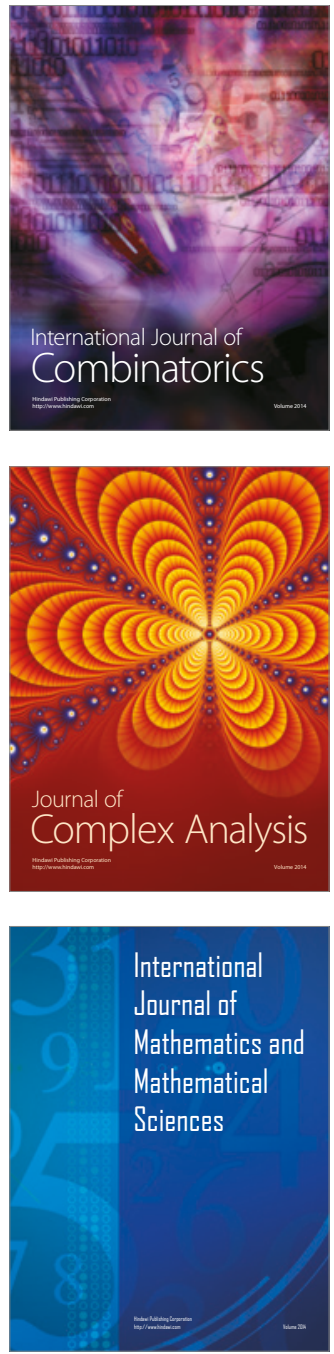
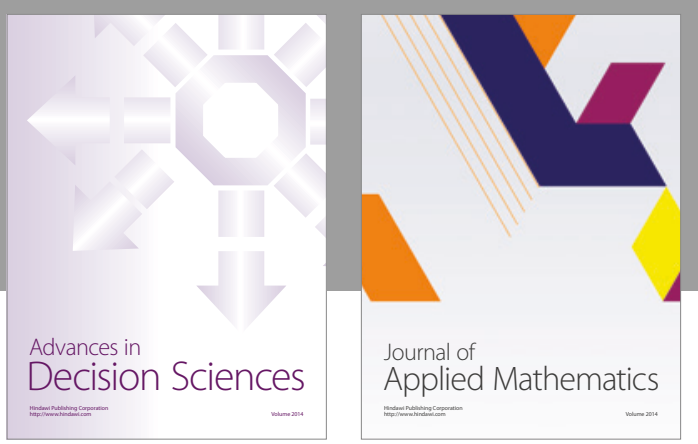

Algebra

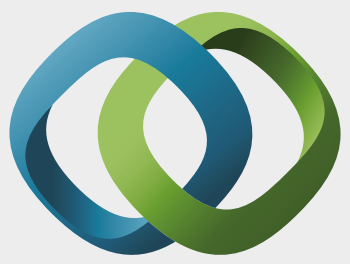

\section{Hindawi}

Submit your manuscripts at

https://www.hindawi.com
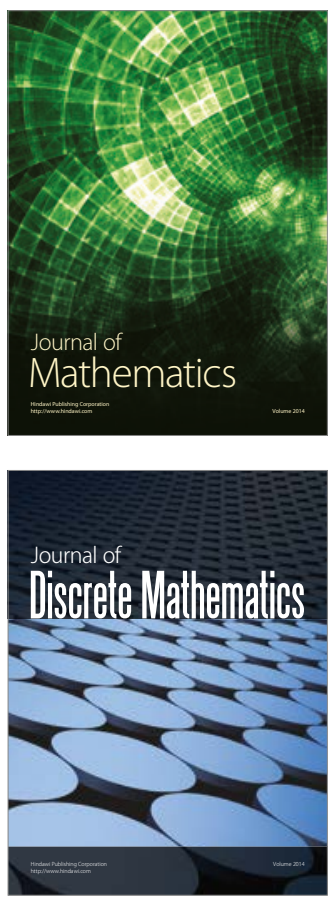

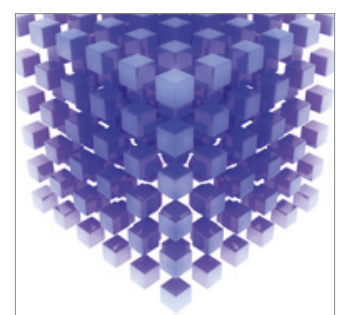

Mathematical Problems in Engineering
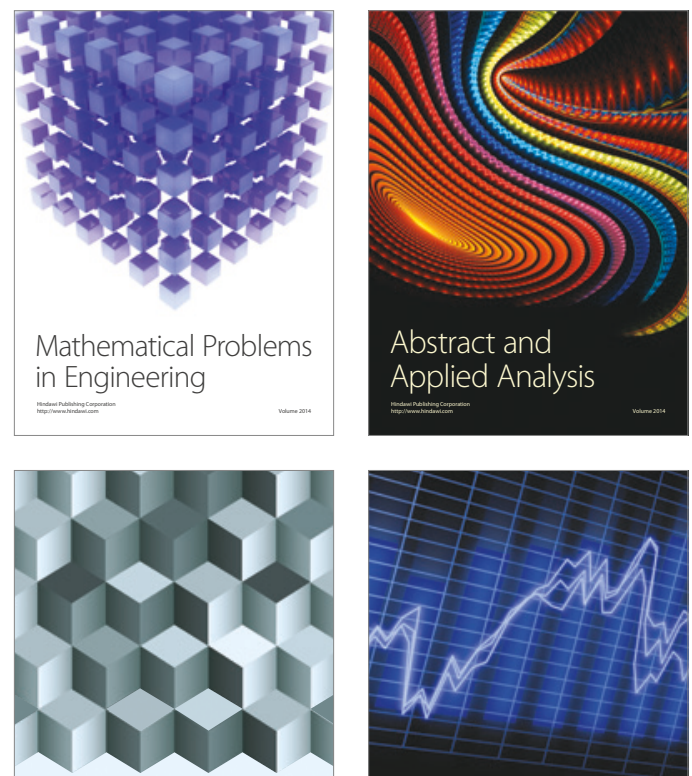

Journal of

Function Spaces

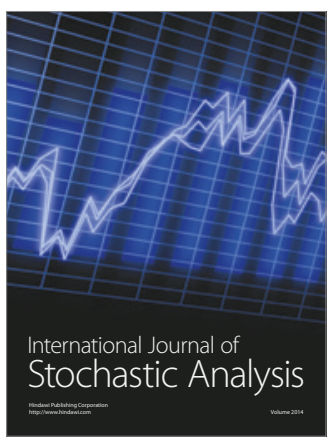

Probability and Statistics
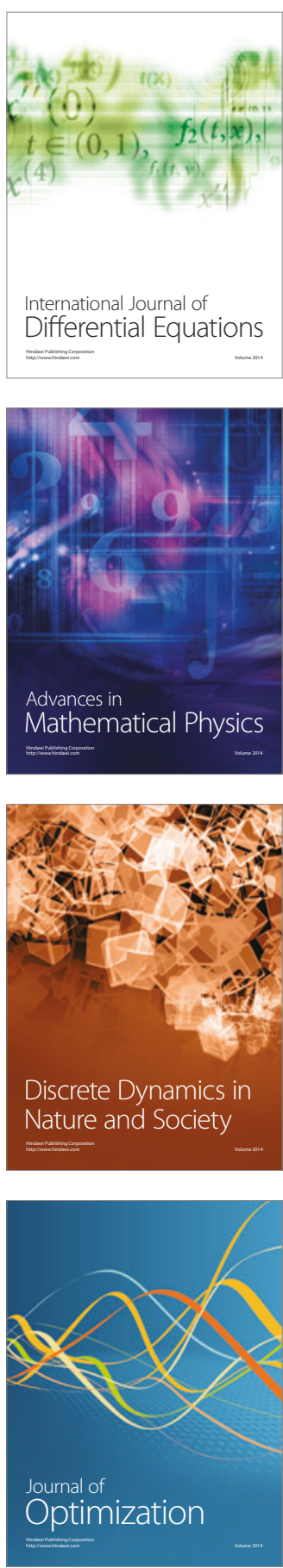